\title{
Preclinical development of highly effective and safe DNA vaccines directed against HPV 16 E6 and E7
}

\author{
Koen Oosterhuis ${ }^{1}$, Peter Öhlschläger ${ }^{2}$, Joost H. van den Berg ${ }^{1,3}$, Mireille Toebes ${ }^{1}$, Raquel Gomez ${ }^{1}$, \\ Ton N. Schumacher ${ }^{1}$ and John B. Haanen ${ }^{1}$ \\ 'Division of Immunology, The Netherlands Cancer Institute, Amsterdam, The Netherlands \\ ${ }^{2}$ Department of Immunology, University of Constance, Konstanz, Germany \\ ${ }^{3}$ Department of Pharmacy and Pharmacology, Slotenvaart Hospital, Amsterdam, The Netherlands
}

To allow vaccination irrespective of HLA type, DNA vaccines encoding full-length antigens are required. However, here, we demonstrate that the immunogenicity of DNA vaccines encoding the full-length human papillomavirus (HPV) type 16 E7 and E6 proteins is highly reduced compared to vaccines encoding only the immunodominant epitope. Furthermore, the low remaining immunogenicity is essentially lost for both E7 and E6 when a nononcogenic "gene-shuffled" variant is utilized. To address these issues, we tested whether alterations in transgene design can restore the immunogenicity of full-length and geneshuffled DNA vaccines. Remarkably, genetic fusion of E7 with tetanus toxin fragment C (TTFC) resulted in a dramatic increase in immunogenicity both for the full-length and the gene-shuffled version of E7. Moreover, the TIFC fusion vaccines were more immunogenic than a vaccine encoding a fusion of E7 and mycobacterial heat shock protein-70, which has recently been tested in a clinical trial. Interestingly, vaccination with these TTFC fusion vaccines also resulted in extremely persistent T-cell responses. The E7-specific CD8 ${ }^{+} T$ cells induced by TTFC fusion vaccines were functional in terms of IFN- $\gamma$ production, formation of immunological memory, in vivo cytolytic activity and tumor eradication. Finally, we show that genetic fusion with TTFC also improves the immunogenicity of a gene-shuffled E6 DNA vaccine. These data demonstrate that genetic fusion with tetanus toxin fragment $C$ can dramatically improve the immunogenicity of full-length and gene-shuffled DNA vaccines. The DNA fusion vaccines developed here will be evaluated for the treatment of HPV-positive carcinomas in future studies.

Persistent infection with "high-risk" human papillomavirus (HPV) genotypes is strongly associated with the development of anogenital cancers. ${ }^{1,2}$ Of the "high-risk" genotypes, HPV 16 alone is known to be responsible for about half of the cervical cancer cases worldwide. ${ }^{3}$ Because persistent expression of the oncogenic HPV proteins E6 and E7 is required for carcinogenesis, these viral antigens are ideal targets for immunotherapeutic interventions. As E6 and E7 are solely expressed intracellularly, such therapeutic interventions should induce cellular immune responses to control existing HPV-induced lesions. ${ }^{3,4}$

DNA vaccination forms an attractive approach for the induction of cellular immune responses as the DNA-encoded

Key words: DNA vaccination, CD8+, T cells, HPV 16, tetanus toxin fragment $\mathrm{C}$, genetic fusion

Additional Supporting Information may be found in the online version of this article

Grant sponsor: Netherlands Organization for Health Research en Development (ZonMw); Grant number: 432-00-001

Correspondence to: John B. Haanen, Division of Immunology, The Netherlands Cancer Institute, Plesmanlaan 121, 1066 CX, Amsterdam, The Netherlands, Tel.: +31-20-5126979, Fax: +31-20-5122057, E-mail: j.haanen@nki.nl antigens are by definition produced intracellularly. Furthermore, DNA vaccines are safe, easy to produce, stable and do not suffer from the drawback of preexisting immunity or induction of antivector immunity. ${ }^{5,6}$ In murine models, numerous DNA vaccines directed against either HPV 16 E6 or E7 have been tested with promising results..$^{7-13}$ However, to date, the clinical translation of these approaches has met little success. ${ }^{14,15}$ Recently, we developed a novel DNA vaccination strategy named DNA tattoo vaccination that can potentially overcome this translational block. This strategy was shown to lead to the rapid induction of cellular immunity when compared to conventional methods of DNA vaccination in mice. ${ }^{16}$ Furthermore, DNA tattooing outperformed classical intramuscular DNA vaccination by 10 - to 100 -fold when tested in non-human primates. ${ }^{17}$ Currently, DNA tattoo vaccination is being evaluated in a Phase I clinical trial for the treatment of melanoma, using a DNA vaccine that was produced in house in a GMP compliant plasmid production facility. ${ }^{18}$

In our current study, we describe the preclinical development of DNA vaccines directed against HPV 16 E6 and E7, aiming for optimal safety and immunogenicity. In earlier work, we have established that DNA vaccines that encode single defined antigen-derived T-cell epitopes are highly immunogenic. ${ }^{19}$ However, the extensive polymorphism of HLA alleles precludes the broader application of such epitopedirected DNA vaccines, and we therefore set out to develop 
effective DNA vaccines that encompass the full epitopeencoding potential of the HPV 16 E6 and E7 proteins. Furthermore, as HPV 16 E6 and E7 are oncogenes through their ability to induce degradation of the tumor suppressors p53 and $\mathrm{pRb}$, respectively, ${ }^{20,21}$ the transforming potential of these genes needs to be eliminated before application in humans. Two strategies have previously been put forward to disrupt the oncogenic potential of E6 and E7 in DNA vaccines. First, point mutations in the pRb-binding site for E7 and in the p53-binding site for E6 can prevent degradation of these targets and thus prevent cellular transformation. ${ }^{11,22}$ Second, a more drastic approach-termed gene shuffling-has been developed recently to prevent the risk of cellular transformation by HPV 16 E7. ${ }^{9,23}$ In this approach, the gene sequence of E7 was taken apart at exactly those positions that are critical for the known transforming properties of the protein, and the resulting fragments were reassembled in a "shuffled" order. To avoid the loss of putative CD8 $+\mathrm{T}$-cell epitopes at the junctions, sequences encoding the nine amino acids at either side of the different junctions in the natural protein were added as an "appendix." As the three-dimensional structure of the resulting protein product will be markedly different from that of the parental protein, it is plausible that for thus shuffled proteins not only the binding to known cellular targets but also interaction with other potential targets $^{20,21,24,25}$ is prevented. Consequently, gene shuffling can be considered a preferred approach from a safety perspective.

Here, we demonstrate that the immunogenicity of DNA vaccines that encode the full-length HPV 16 E6 or E7 proteins is highly reduced when compared to vaccines in which only the immunodominant epitope is present. Furthermore, this low remaining immunogenicity is essentially lost when the preferred shuffled E6 and E7 vaccine formats are utilized. We subsequently demonstrate how DNA vaccines with a superior capacity for $\mathrm{CD} 8{ }^{+} \mathrm{T}$-cell priming can be generated through the genetic fusion of either full-length or gene-shuffled HPV genes with domain 1 of tetanus toxin fragment C (TTFC). Collectively, these experiments define the transgene formats for HPV $16 \mathrm{E} 6$ and E7 DNA vaccines for use in an upcoming Phase I clinical trial.

\section{Material and Methods \\ Mice}

C57BL/6 mice (6-10 weeks) were obtained from the experimental animal department of The Netherlands Cancer Institute (Amsterdam, The Netherlands). All experiments were performed in accordance with institutional and national guidelines and were approved by the Experimental Animal Committee of The Netherlands Cancer Institute and in accordance with institutional and national guidelines.

\section{DNA vaccines}

DNA vaccines based on HPV 16 E6 and E7 genes were generated by the introduction of target genes or gene fragments into pVAX 1 (Invitrogen, Carlsbad, CA). The generation of
GFP-E7 ${ }_{49-57}$ has been described elsewhere. ${ }^{26}$ GFP-E6 $_{48-57}$ encodes the immunodominant $\mathrm{H}-2 \mathrm{~K}^{\mathrm{b}}$-restricted epitope EVYDFAFRDL as a genetic fusion with GFP and was constructed in an analogous manner as GFP-E7 ${ }_{49-57}$, in between the BamHI and Not I sites of pVAX. E7WT, E7GGG, ${ }^{11}$ E6WT and $\mathrm{E}_{6 \mathrm{GG}}{ }^{22}$ were obtained from GeneArt (Hilden, Germany), with codon optimization for expression in human cells, and were all cloned between the HindIII and XbaI sites of pVAX. The generation of E7SH has been described elsewhere, ${ }^{23}$ and E6SH was constructed in a similar fashion. In brief, E6 was cut at positions corresponding to aa 31/32, aa $64 / 65$, aa $104 / 105$ and aa $137 / 138$; the resulting five segments were reassembled in the order ADCBE, and the original junctions destroyed by the dissection were added as an "appendix." The design of E6SH is shown in Supporting Information Figure 1. For our current study, both E6SH and E7SH were cloned between HindIII and XbaI sites of pVAX. The TTFC fusions, TTFC-E7WT, TTFC-E7GGG, TTFC-E7SH and TTFC-E6SH, were generated by $\mathrm{C}$-terminal fusion of the gene of interest to TTFC domain 1 through PCR. All PCR products were cloned into the HindIII and XbaI sites of pVAX. pNGVL4a-sig/E7(detox)HSP70 ${ }^{15}$ was a kind gift from T.C. Wu. Sequences were confirmed by sequence analysis. Plasmids were expressed and amplified in E. coli DH5 $\alpha$ and were purified using an endotoxin-free DNA purification kit (Qiagen, Hilden, Germany). DNA vaccines for intradermal tattoo application were dissolved in water for injections (Aqua B. Braun, Melsungen, Germany).

\section{Tattoo vaccination}

Intradermal DNA tattoo vaccination was performed at Day 0, 3 and 6 , as described previously, with minor modifications. ${ }^{16}$ One day before the first DNA tattoo, the hair on the hind leg was removed using depilating cream (Veet ${ }^{(1)}$, Reckitt Benckiser, Hull, UK). On the day of vaccination, mice were anesthetized, and $10 \mu \mathrm{l}$ of a $2 \mu \mathrm{g} / \mu \mathrm{l}$ DNA solution in water was applied to the hairless skin of the hind leg. The DNA vaccine was applied with a Permanent Make Up tattoo machine (kindly provided by MT Derm GmbH, Berlin, Germany), using a sterile disposable nine-needle bar with a needle depth of $1 \mathrm{~mm}$ and oscillating at a frequency of $100 \mathrm{~Hz}$ for $30 \mathrm{sec}$.

\section{Detection of HPV-specific T cells in peripheral blood}

Peripheral blood cells were obtained via tail bleeding, and erythrocytes were removed by incubation in erythrocyte lysis buffer $\left(155 \mathrm{mM} \mathrm{NH}_{4} \mathrm{Cl}, 10 \mathrm{mM} \mathrm{KHCO}_{3}\right.$ and $0.1 \mathrm{mM}$ EDTA, $\mathrm{pH}$ 7.4) on ice. The cells were subsequently stained in FACS buffer ( $1 \times$ PBS, $0.5 \%$ BSA and $0.02 \%$ sodium azide) with allophycocyanin (APC)-conjugated anti-CD8a mAb (BD Pharmingen, San Jose, CA) plus phycoerythrin (PE)-conjugated $\mathrm{H}-2 \mathrm{D}^{\mathrm{b}} \mathrm{E}_{49-57}$ or $\mathrm{H}-2 \mathrm{~K}^{\mathrm{b}} \mathrm{E6}_{48-57}$ tetramers for 15 min at $20^{\circ} \mathrm{C}$. Subsequently, cells were washed two times in FACS buffer before analysis. Live cells were selected based on PI exclusion. MHC tetramers were produced by UV-induced peptide exchange, as described previously. ${ }^{27}$ 

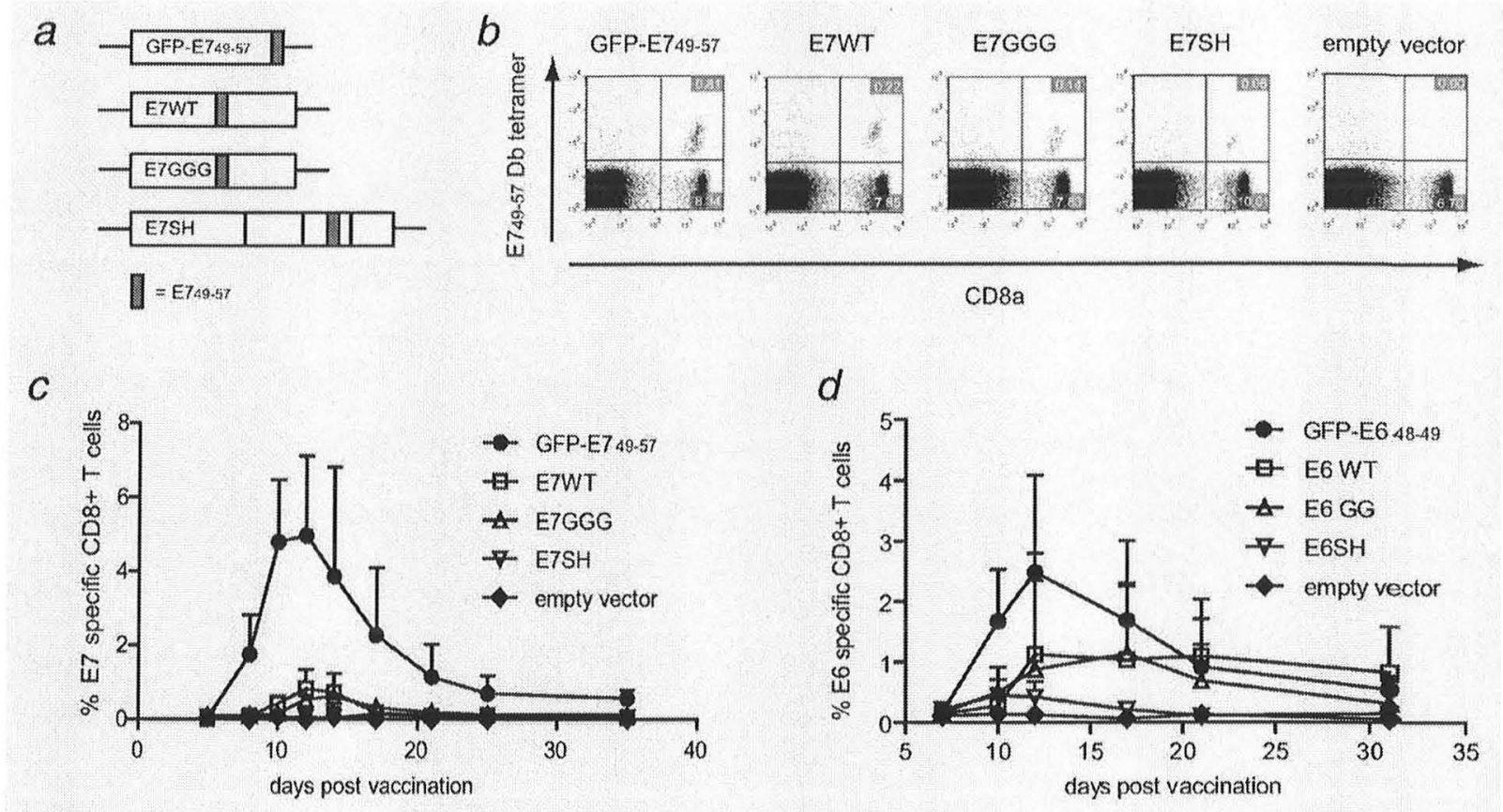

Figure 1. Transgene design and immunogenicity of HPV $16 \mathrm{E}$-and E6-encoding DNA vaccines. C57BL/6 mice ( $n=5$ per group) were immunized by DNA tattoo vaccination on Day 0,3 and 6, and peripheral blood was analyzed for antigen-specific CD8 ${ }^{+} \mathrm{T}$ cells by MHC tetramer staining. (a) Schematic representation of the E7-directed DNA vaccines. GFP-E7 ${ }_{49-57}$ encodes the immunodominant $\mathrm{H}-2 \mathrm{D}^{\mathrm{b}}$ restricted epitope of HPV $16 \mathrm{E7}_{49-57}$ as a C-terminal fusion with eGFP. E7GGG encodes an E7 variant with three point mutations in the pRbbinding domain: D21G, C24G and E26G. ${ }^{11}$ E7SH encodes a "gene-shuffled" variant of E7. ${ }^{23}$ HPV E6-directed DNA vaccines with corresponding names were constructed in an identical fashion (not shown). (b) Representative dot plots of MHC tetramer stainings at the

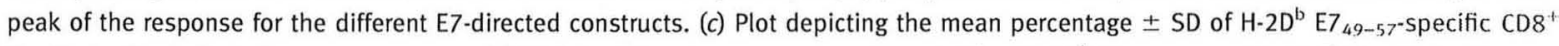
$\mathrm{T}$ cells for the indicated groups over time. (d) Plot depicting the mean percentage $\pm \mathrm{SD}$ of $\mathrm{H}-2 \mathrm{~K}^{\mathrm{b}} \mathrm{E} 6_{48-57^{-}}$-specific $\mathrm{CD} 8^{+} \mathrm{T}$ cells for the indicated groups over time.

IFN- $\gamma$ assays were performed using the BD Cytofix/Cytoperm kit (Becton Dickinson Sciences, Franklin Lakes, NJ) according to the manufacturer's protocol. Peripheral blood cells were stimulated for $4 \mathrm{hr}$ at a $1 \mu \mathrm{g} \mathrm{E7}_{49-57}$ peptide (RAHYNIVTF) concentration and subsequently stained using PE-conjugated anti-INF- $\gamma$ mAb (BD Pharmingen, San Diego, $\mathrm{CA}$ ) and APC-conjugated anti-CD8a mAb (BD Pharmingen). All samples were analyzed on a FACScalibur (Becton Dickinson) using Flow-Jo software (Three Star, Ashland, USA) for data analysis.

\section{In vivo cytotoxicity assay}

The capacity to kill peptide-loaded target cells in vivo was assayed as described previously. ${ }^{28}$ Briefly, splenocytes from naive mice were labeled with either $0.1 \mu \mathrm{M}$ CFSE (low) or 1 $\mu \mathrm{M}$ CFSE (high). The cells labeled with $1 \mu \mathrm{M}$ CFSE were subsequently pulsed with $10 \mu \mathrm{M}$ RAHYNIVTF peptide for 1 $\mathrm{hr}$ at $37^{\circ} \mathrm{C}$, and the cells labeled with $0.1 \mu \mathrm{M}$ CFSE were pulsed with a control (influenza A-derived ASNENMDAM) peptide. After peptide loading, cells were washed three times and subsequently injected into mice in a 1:1 ratio for a total of $2 \times 10^{6}$ cells per mouse. After $20 \mathrm{hr}$, spleen cells were iso- lated, and the ratio of $\mathrm{CFSE}_{\text {high }} / \mathrm{CFSE}_{\text {low }}$ cells was determined by flow cytometry. The percentage antigen-specific cytotoxicity was determined as follows: $100-\left(\left[\% \mathrm{CFSE}_{\text {high }}\right.\right.$ in vaccinated $/ \% \mathrm{CFSE}_{\text {low }}$ in vaccinated $) /\left(\% \mathrm{CFSE}_{\text {high }}\right.$ in naive $\%$ $\mathrm{CFSE}_{\text {low }}$ in naive)] * 100).

\section{TC-1 tumor challenge}

C57BL/6 mice were injected subcutaneously with $1 \times 10^{5}$ TC-1 tumor cells that express both HPV 16 E6 and E7. ${ }^{29}$ DNA tattoo vaccination was subsequently performed on Day 3,6 and 9 after tumor challenge. Tumor growth was monitored one to three times per week using caliper measurements in two dimensions. The volume of the tumors was calculated as follows: volume $=\left(\right.$ width $^{2} \times$ length $) / 2 .{ }^{30}$ Mice were sacrificed when the tumor diameter reached $15 \mathrm{~mm}$ or when the tumor volume exceeded $1,000 \mathrm{~mm}^{3}$.

\section{Statistical analysis}

Statistical analysis was performed using a Student's $t$-test. A $p$ value $<0.05$ was considered to be significant (two tailed). All findings were confirmed in at least one additional independent experiment. 


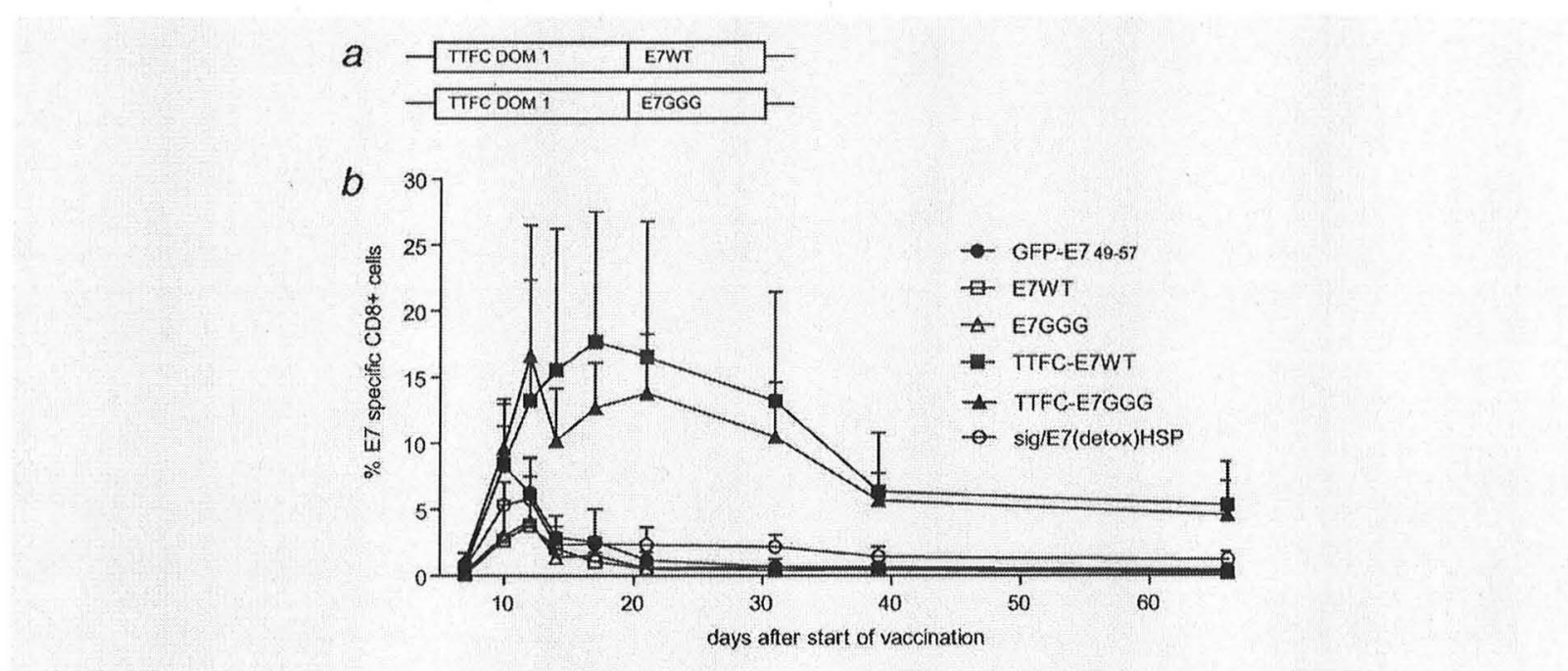

Figure 2. TTFC fusion enhances DNA vaccine immunogenicity. C57BL/6 mice ( $n=5$ per group) were immunized by DNA tattoo vaccination on Day 0, 3 and 6, and peripheral blood was analyzed for antigen-specific $\mathrm{CD}^{+} \mathrm{T}$ cells by MHC tetramer staining. (a) Schematic representation of E7 variants that were expressed as C-terminal fusions with tetanus toxin fragment $\mathrm{c}$ domain 1 (TTFC). ${ }^{31}$ (b) Plot depicting

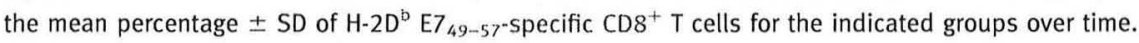

\section{Results}

Immunogenicity of HPV 16 E7 and E6 DNA vaccines is highly dependent on transgene design

We first compared the immunogenicity of the immunodominant HPV $16 \mathrm{E}_{49-57}$ epitope when either present within the full-length $\mathrm{E} 7$ gene or as a C-terminal fusion of the single epitope with GFP (see Figs. 1 $a-1 c$ ). Consistent with earlier results, ${ }^{16,19}$ ex vivo $\mathrm{MHC}$ tetramer staining of peripheral blood of mice that had received a DNA vaccine encoding GFP-E7 ${ }_{49}$ 57 demonstrated that this epitope-directed vaccine was highly immunogenic (peak T-cell response of $4.97 \% \pm 2.15 \%$ ). In contrast, immune responses against the same epitope remained low in mice that were vaccinated with the E7WT-encoding vaccine $\left(0.83 \% \pm 1.14 \%, p<0.01\right.$ versus $\left.\mathrm{GFP}_{-\mathrm{E} 7}{ }_{49-57}\right)$. The immunogenicity of the full-length E7 was not altered by the introduction of point mutations, as immunogenicity of E7GGG was equally low $(0.63 \% \pm 0.66 \%, p<0.01$ versus GFP-E7 $49-57)$. However, the immune responses elicited by a DNA vaccine in which the same epitope was present in the "shuffled" version of the E7 gene (E7SH) were not significantly different from the background responses detected in mockvaccinated animals $(p=0.57)$. Similar to what was observed for E7, immune responses induced by a shuffled HPV E6 DNA vaccine $(\mathrm{E} 6 \mathrm{SH})$ were close to background and substantially lower than those induced by E6WT, E6GG and, in particular, GFP-E6 ${ }_{48-57}$ DNA vaccines (5.8-fold lower, $p<0.05$ ) (Fig. $1 d$ ). Taken together, these results demonstrate that the context in which a defined HPV $16 \mathrm{E} 7$ or $\mathrm{E} 6 \mathrm{CD}^{+}{ }^{+} \mathrm{T}$-cell epitope is delivered strongly influences its immunogenicity. Importantly, the poor performance of the full-length or shuffled versions necessitated the optimization of the DNA vaccine design before moving to clinical evaluation.
Fusion of full-length E7 with TTFC domain 1 results in a dramatic increase in $\mathrm{CD8}^{+} \mathrm{T}$-cell responses

As $\mathrm{CD}^{+}$T-cell responses to the gene-shuffled DNA vaccines were essentially undetectable, we first focused on improving the immunogenicity of the wild-type and pointmutated versions, choosing HPV $16 \mathrm{E} 7$ as a model vaccine. Prior work by Stevenson and coworkers has demonstrated that fusion of antigenic peptides with the C-terminus of TTFC domain 1 (hereafter referred to as TTFC) enhances $\mathrm{CD}^{++} \mathrm{T}$-cell responses against these peptides. ${ }^{31}$ To evaluate whether this strategy would also be successful for the fulllength $\mathrm{E} 7$ protein, we generated DNA vaccines consisting of C-terminal fusions of E7WT and E7GGG with TTFC (Fig. 2a). Vaccination of mice with TTFC-E7WT and TTFCE7GGG resulted in $\mathrm{E}_{49-57}$-specific $\mathrm{CD}^{+} \mathrm{T}$-cell responses that were markedly increased relative to those induced by E7WT, with mean peak CD8 ${ }^{+}$T-cell frequencies of $17.6 \% \pm$ $9.83 \%$ and $16.6 \% \pm 9.85 \%$, respectively (Fig. $2 b$ ). Importantly, the responses induced by the TTFC-fusion vaccines were also markedly higher than those induced by sig/ E7(detox)HSP (mean peak CD8+ T-cell frequencies of $5.79 \% \pm 3.15 \%)$, which has recently been tested in a clinical trial. ${ }^{15}$ Surprisingly, fusion to TTFC did not only increase the peak height of the CTL response but also caused a marked change in response kinetics. Specifically, although classical vaccine-induced cellular immune responses (as induced by all other vaccine formats tested) are characterized by a rapid contraction after the peak of the T-cell response, tattoo vaccination with TTFC-E7 DNA vaccines induced $\mathrm{CD}^{+} \mathrm{T}$-cell responses that remained near constant for about 3 weeks after the peak of the $\mathrm{CD}^{+}$T-cell response was reached (Fig. 2b). Furthermore, also in the months 

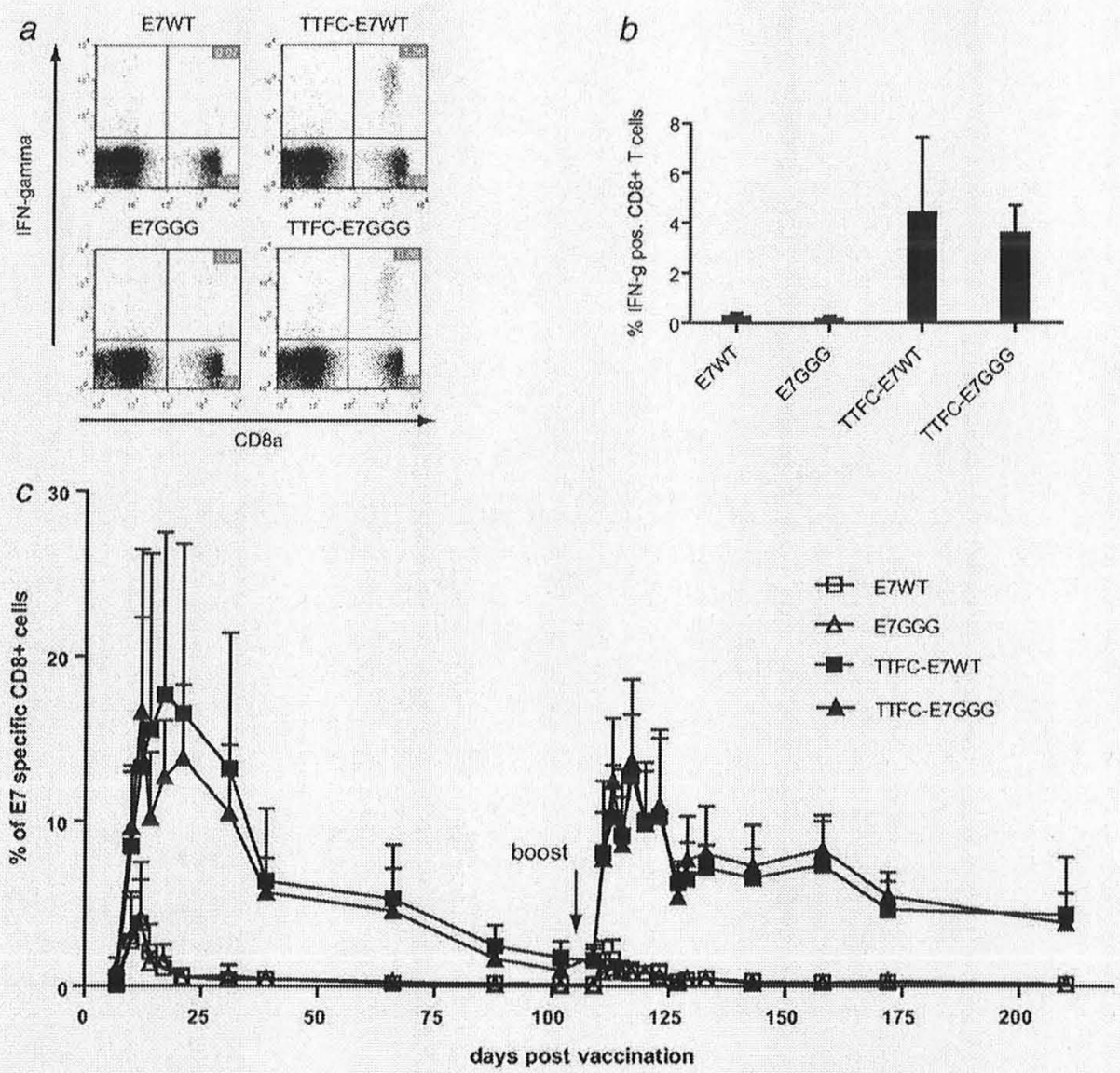

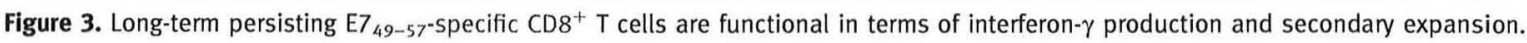
Interferon- $\gamma$ production of peripheral blood $\mathrm{CDB}^{+}$cells from indicated groups of the experiment displayed in Figure 2 was assayed 4 weeks after start of vaccination. (a) Representative dot plots of IFN- $\gamma$ staining of the indicated groups. (b) Bar diagram showing the mean percentage \pm SD of interferon- $\gamma$-positive CD8 $T$ cells after 4-hr stimulation with the E7 ${ }_{49-57}$ peptide. (c) Secondary T-cell responses of TFC-E7WT- or TTFC-E7GGG-vaccinated mice. Mice from the experiment displayed in Figure 2 were boosted with a single homologous DNA tattoo vaccination at the indicated time point. Peripheral blood was analyzed for antigen-specific $\mathrm{CD} 8^{+} \mathrm{T}$ cells by $\mathrm{MHC}$ tetramer staining. The mean percentage $\pm \mathrm{SD}$ of $\mathrm{H}-2 \mathrm{D}^{\mathrm{b}} \mathrm{E} 7_{49-5} \gamma_{-5}$-5ecific $\mathrm{CD}^{+} \mathrm{T}$ cells for the indicated groups is displayed over time.

following vaccination, marked DNA vaccine-induced T-cell responses remained detectable directly ex vivo.

\section{Functional characterization of TTFC E7 induced long-term persisting cells}

As the kinetics of the E7-specific $\mathrm{CD}^{+}$T-cell responses induced by TTFC-E7 vaccines were so markedly different from classical vaccine-induced $\mathrm{CD}^{+}{ }^{+} \mathrm{T}$-cell responses, we investigated the functional properties of these cells. First, the ability of $\mathrm{CD}^{+} \mathrm{T}$ cells to produce IFN- $\gamma$ upon $\mathrm{E}_{49-57}$ peptide stimulation was tested by intracellular cytokine staining of peripheral blood samples taken from DNA tattoo-vaccinated mice (Fig. 3 ). Four weeks after vaccination, the production of IFN- $\gamma$ could be detected in $4.4 \% \pm 3.03 \%$ and $3.6 \% \pm 1.14 \%$ of $\mathrm{CD}^{+} \mathrm{T}$ cells of TTFC-E7WT- and TTFC-E7GGG-vaccinated mice, respectively. In contrast, essentially no IFN- $\gamma$ production above background could be observed in samples taken from E7WTand E7GGG-vaccinated mice. Second, the ability of the vaccination-induced $\mathrm{T}$-cell pool to respond to secondary antigen encounter was evaluated by a single homologous booster vaccination 3 months after priming (Fig. 3c). Peak secondary T-cell responses were comparable in size to the primary response for both TTFC-E7WT and TTFC-E7GGG. In contrast, in E7WTand E7GGG-vaccinated mice, secondary T-cell responses were markedly reduced relative to the primary response. As a result, the differences between E7WT and TTFC-E7WT (7.7-fold; $p$ $<0.05)$ and E7GGG and TTFC-E7GGG (12.2-fold; $p<0.001)$ were even more pronounced during the secondary response. As a third and final test for T-cell functionality of the longterm persisting HPV E7-specific $\mathrm{CD}^{+} \mathrm{T}$ cells, the ability to kill peptide-loaded target cells was tested in an in vivo cytolytic assay 6 weeks after priming with either TTFC-E7GGG or 

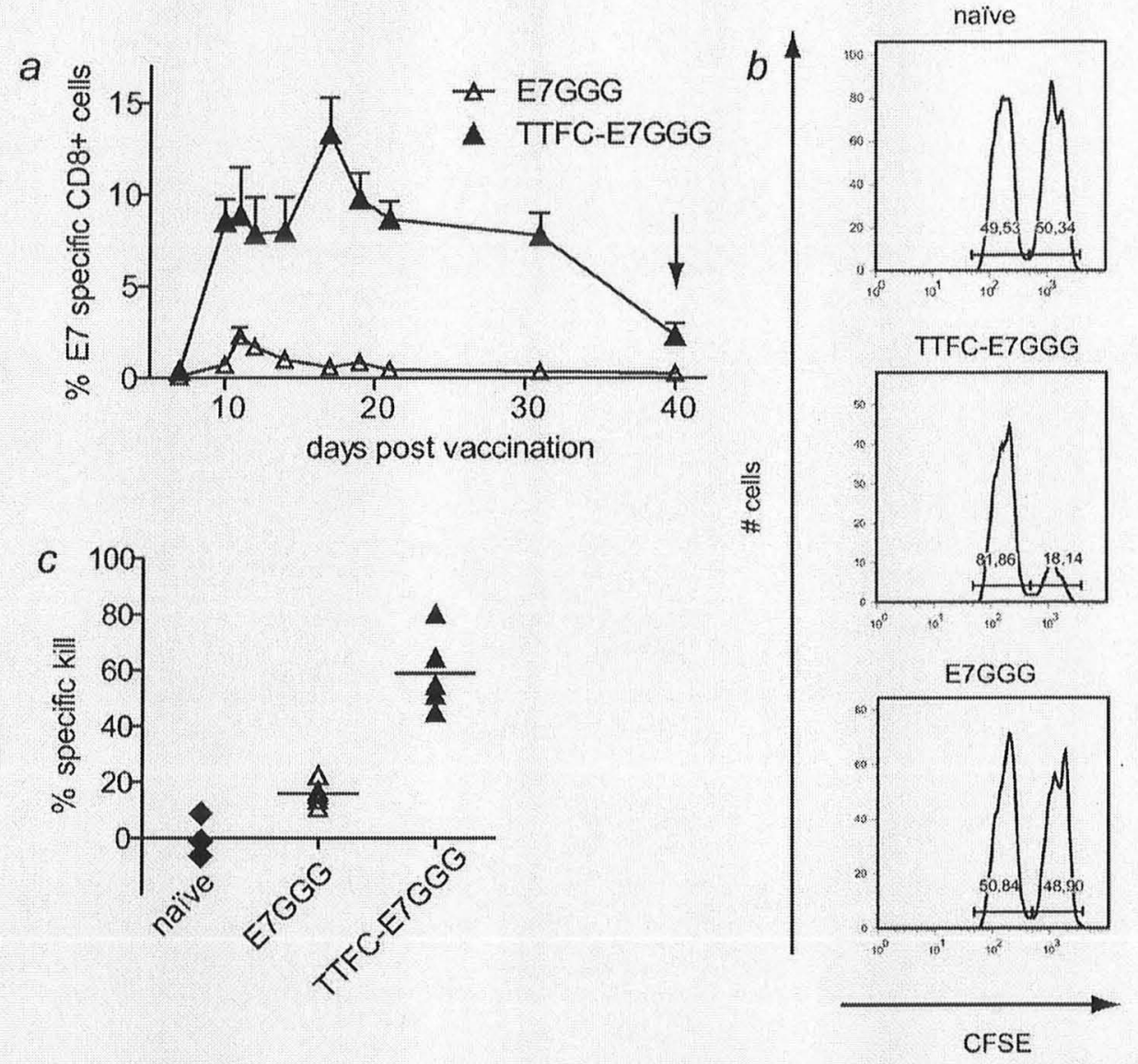

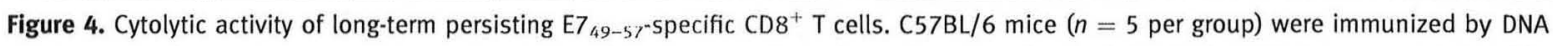
tattoo vaccination on Day 0,3 and 6 , and peripheral blood was analyzed for antigen-specific CD8 ${ }^{+} \mathrm{T}$ cells by MHC tetramer staining. (a) Plot depicting the percentage $\pm S D$ of $H-2 D^{\mathrm{b}} E 7_{49-57-5 p e c i f i c ~} \mathrm{CDB}^{-} \mathrm{T}$ cells over time. The arrow indicates the time point at which mice were injected with peptide-loaded target cells. (b) Representative histograms displaying the ratio of target cells pulsed with specific peptide (CFSE high) or irrelevant peptide (CFSE low) $20 \mathrm{hr}$ after injection. (c) Plot depicting the percentage of specific kill of E7 ${ }_{49-57}$-pulsed target cells. Displayed are the individual values and the mean of each group.

E7GGG (Fig. 4). In TTFC-E7GGG-vaccinated mice, 59.1\% \pm $13.7 \%$ specific lysis of target cells was observed compared to only $15.9 \% \pm 4.3 \%$ in E7GGG-vaccinated mice $(p=0.0013)$. Taken together, these results demonstrate that DNA tattoo vaccination of mice with TTFC-E7 fusion vaccines induces superior T-cell reactivity compared to $\mathrm{E} 7$ vaccines as revealed by both antigen-specific $\mathrm{T}$-cell numbers and their function.

\section{TTFC fusion also enhances the immunogenicity of the E7SH and E6SH DNA vaccines}

Because of the perceived greater safety of shuffled HPV 16 E6 and E7 genes when compared to point-mutated HPV 16 E6/E7 genes, we evaluated whether the immunogenicity of the very weakly immunogenic shuffled DNA vaccines could also be enhanced by TTCF fusion. As shown in Figure $5 a$, the use of TTFC-E7SH resulted in a strong E7-specific CD8 ${ }^{+}$ T-cell response with an 11.7 -fold $(p<0.01)$ difference at the peak of the response compared to E7SH. Moreover, both the magnitude and persistence of the primary and secondary $\mathrm{CD} 8^{+} \mathrm{T}$-cell response induced by TTFC-E7SH were comparable to that induced by vaccination with TTFC-E7WT. Evaluation of the magnitude of $\mathrm{CD}^{+}$T-cell responses induced by TTFC-E6SH relative to those induced by $\mathrm{E} 6 \mathrm{SH}$ (Fig. $5 b$ ) revealed a modest but nonsignificant increase in primary T-cell responses (3.0-fold; $p=0.27$ ). The delayed contraction of the vaccination-induced immune response, observed for TTFC-E7 fusions, was not seen for TTFCE6SH. However, following homologous boost vaccination, E6-specific CD8 ${ }^{+}$T-cell responses in TTFC-E6SH-vaccinated mice were markedly higher than those in $\mathrm{E} 6 \mathrm{SH}$-vaccinated mice (14.6-fold at the peak of the response, $p<0.01$ ). Taken together, these results demonstrate that TTFC fusion enhances the immunogenicity of both E7SH and E6SH DNA vaccines. 

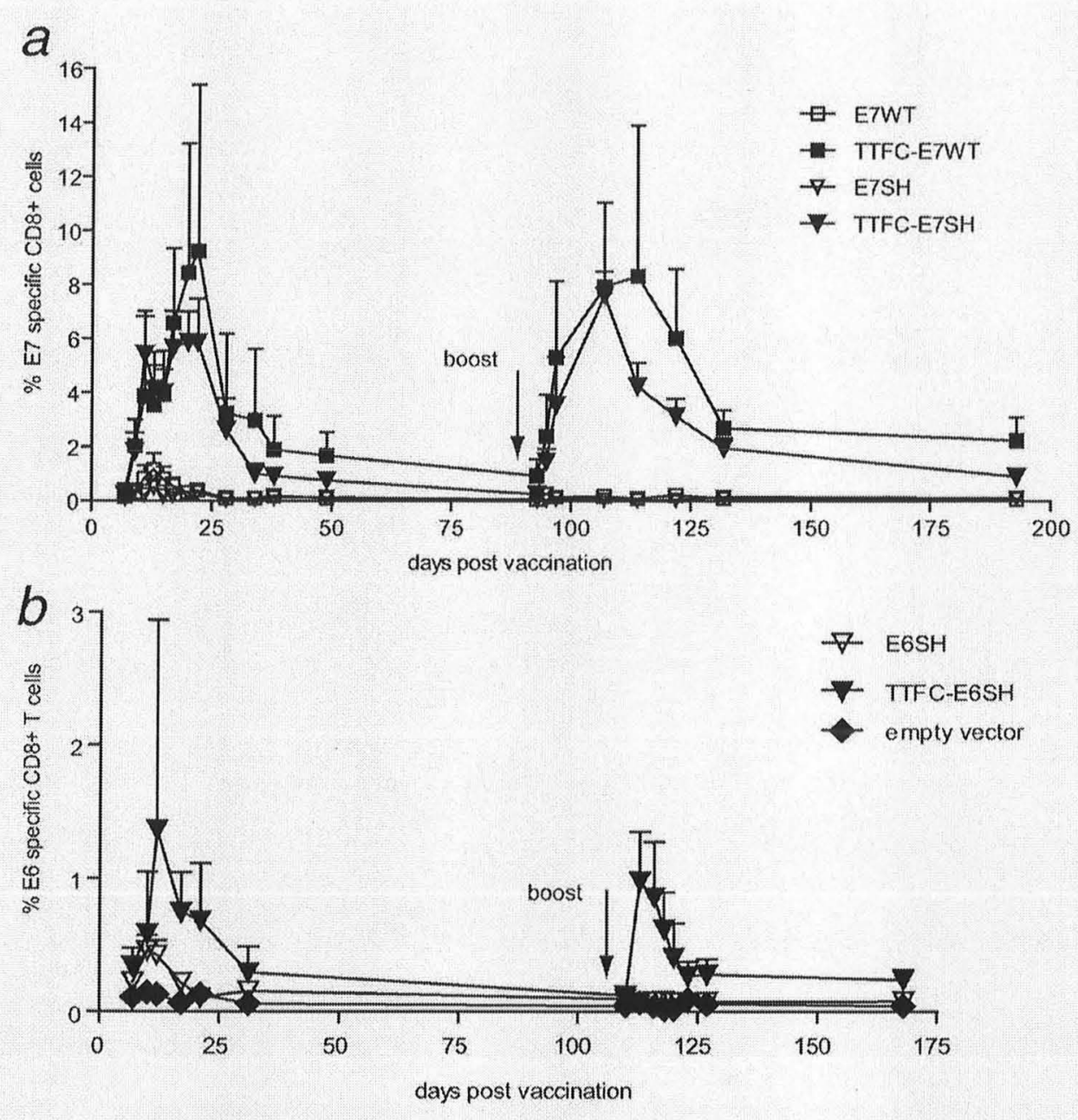

Figure 5. TFC fusion enhances the immunogenicity of E7SH and E6SH. C57BL/6 mice ( $n=5$ per group) were immunized by DNA tattoo vaccination on Day 0,3 and 6 and boosted with a single homologous tattoo vaccination at the indicated time point. Peripheral blood was analyzed for antigen-specific $\mathrm{CD} 8^{+} \mathrm{T}$ cells by MHC tetramer staining. (a) Plot depicting the mean percentage $\pm \mathrm{SD}$ of $\mathrm{H}-2 \mathrm{D}^{\mathrm{b}} \mathrm{E} 7_{49-57^{-S}}$ Specific $\mathrm{CD}^{+} \mathrm{T}$ cells for the indicated groups is displayed over time. (b) Plot depicting the mean percentage $\pm \mathrm{SD}$ of $\mathrm{H}-2 \mathrm{~K}^{\mathrm{b}} \mathrm{Eb}_{48-57^{-5}} \mathrm{Secific}^{\mathrm{CD} 8^{+} \mathrm{T}}$ cells for the indicated groups over time.

\section{Comparing the antitumor effect of E7SH and TTFC-E7SH DNA vaccines}

To evaluate whether the difference in immunogenicity resulting from the TTFC fusion translated into a measurable difference in the ability of vaccine-induced $\mathrm{T}$ cells to eradicate HPV 16 E6/E7-transformed tumors, mice were inoculated subcutaneously with $1 \times 10^{5} \mathrm{TC}-1$ tumor cells. At Day 3 post-tumor cell injection, at which point most of the animals carry palpable tumors, tattoo vaccination with E7SH, TTFC-E7SH or a control DNA vaccine was initiated. In the animals treated with empty vector, E7-specific T-cell responses remained below the level of detection indicating that the TC-1 tumor itself is not immunogenic (Fig. 6a). Also, in animals treated with the E7SH DNA vaccine, E7-specific T-cell responses remained close to background, and tumor outgrowth and survival were indistinguishable between the two groups. In contrast, in animals treated with the TTFC-E7SH fusion vaccine, a robust $\mathrm{E} 7$-specific $\mathrm{T}$-cell response emerged $(26.7 \% \pm 20.6 \%)$. This $\mathrm{T}$-cell response was accompanied by tumor regression and resulted in a prolonged survival for all mice (Figs. $6 b$ and $6 c$ ). In spite of the high frequencies of E7-specific CD $\$ \mathrm{~T}$ cells in TTFC-E7SH-vaccinated mice, tumors did eventually recur in $60 \%$ of the mice, indicating that the TC-1 tumors can grow out in the face of an ongoing high-level E7-specific T-cell response. This immune evasion by TC- 1 tumors has been described previously and has been attributed to both mutation of the immunodominant epitope and increased expression of vascular cell adhesion molecule- 1 on the tumor cells. ${ }^{32,33}$ In 

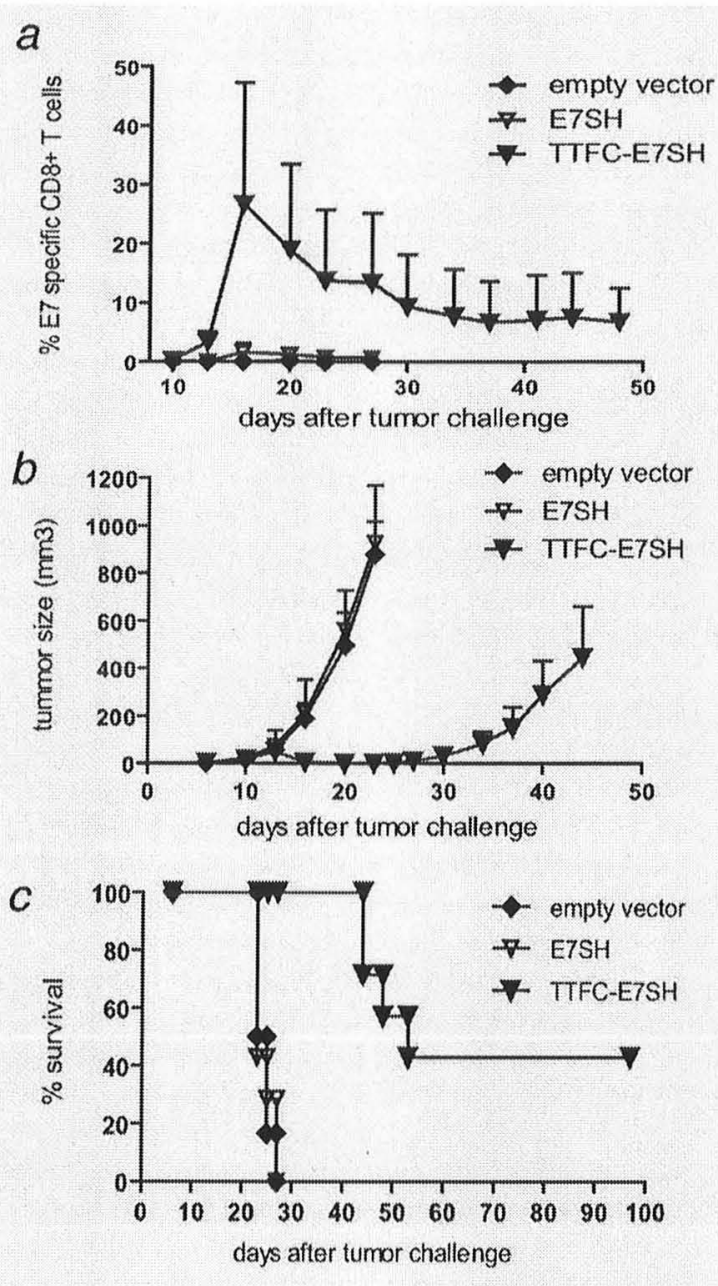

Figure 6. Tumor regression by vaccination with the TTFC-E7SH fusion vaccine. C57BL/6 mice ( $n=5-7$ per group) were injected with $1 \times 10^{5}$ TC-1 tumor cells on Day 0 . Subsequently, mice were immunized by DNA tattoo vaccination on Day 3, 6 and 9 after tumor challenge with the indicated vaccines. Tumor sizes were determined by caliper measurements two to three times weekly. Peripheral blood was analyzed for antigen-specific $\mathrm{CD} 8^{+} \mathrm{T}$ cells by MHC tetramer staining. (a) Plot depicting the mean percentage \pm

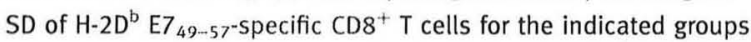
over time. (b) Plot depicting the mean tumor size $\pm \mathrm{SD}\left(\mathrm{mm}^{3}\right)$ for the indicated groups over time. (c) Plot depicting the percentage survival for the indicated groups over time.

summary, the increased immunogenicity of the shuffled E7 DNA vaccine obtained by TTFC fusion resulted in a highly reduced tumor outgrowth and a significant increase in median survival $(p<0.002$; log-rank test).

\section{Discussion}

The aim of our study was to design safe and highly immunogenic DNA vaccines that encode the full-length HPV 16 E6 and
E7 oncogenes. E6- and E7-encoding genetic vaccines thus far used in clinical trials contain point mutations that avoid binding of the encoded proteins to p53 and pRB, respectively. ${ }^{7,34}$ However, it is well recognized that both HPV 16 E6 and E7 also interact with other cellular targets than p53 and pRB, and that these interactions may play an additional role in cellular transformation. ${ }^{1,20,21,24,25,35}$ Because of this concern, the use of E6 and E7 vaccine formats in which the potential of E6 and E7 to interact with cellular targets is altered in a more drastic manner appears preferable. Therefore, we aimed to develop DNA vaccines that encode gene-shuffled variants of E6 and E7. Prior studies have already demonstrated the loss of oncogenic potential of the shuffled variant of E7 in in vitro assays. ${ }^{9,23}$ Likewise, shuffled E6 shows a complete lack of transforming potential (Öhlschlager et al., unpublished observations).

Although shuffled E6 and E7 genes therefore appear suitable for use in genetic vaccines from a safety perspective, we here observed that the immunogenicity of E7SH and E6SH upon DNA tattoo is strongly reduced when compared to both the unmodified and the point-mutated variants of E6 and E7. How can the low immunogenicity of E6SH and E7SH be explained? A possible explanation for the reduced immunogenicity of these shuffled gene products is that these proteins are misfolded and hence rapidly degraded. Prior work has demonstrated that cross presentation of MHC class I-restricted epitopes is biased toward epitopes that accumulate in the antigen-producing cell, ${ }^{36,37}$ and induction of $\mathrm{CD}^{+}$T-cell responses upon DNA tattoo administration is known to depend at least partially on cross presentation. ${ }^{38}$ In further support of this, we have previously demonstrated that in vivo antigen stability and immunogenicity of DNA vaccineencoded antigens that are introduced via intradermal DNA tattooing are correlated, and that the destabilization of a model vaccine via an approach very similar to gene shuffling also resulted in the loss of vaccine immunogenicity. ${ }^{38}$ As a second potential explanation, the gene shuffling procedure could conceivably disrupt $\mathrm{CD} 4^{+} \mathrm{T}$-cell epitopes present within the parental protein. However, as a deleterious effect of gene shuffling is observed for both E6 and E7, and as the regions that encompass the sites at which the parental protein is taken apart are rescued in the "appendix," this explanation seems less likely. It has to be noted that the immunogenicity of (nonfused) E7SH and E7WT was comparable in a previous report. ${ }^{23}$ Potentially, differences in the route of administration (intramuscular injection versus intradermal tattoo) or number of administrations (single injection versus three DNA tattoo applications) could influence the immunogenicity of shuffled DNA vaccines. Regardless of this, from the current analyses, it is apparent that for the planned clinical application, i.e., intradermal DNA tattoo, gene shuffling is highly detrimental to the immunogenicity of both HPV E6 and E7.

We and others have previously demonstrated that DNA vaccines can be improved by fusing genes encoding an antigen or an epitope of interest to that of a carrier protein. ${ }^{7,8,12,19}$ Here, we demonstrate for both E6SH and E7SH 
that their immunogenicity can be fully remedied by genetic linkage to TTFC, priorly developed as a fusion partner in DNA vaccines by the group of Stevenson. ${ }^{6,39}$ In these studies, the optimal configuration for the induction of $\mathrm{CD}^{+} \mathrm{T}$-cell immunity consisted of a C-terminal fusion of a minimal epitope with domain 1 of FrC (here referred to as TTFC for simplicity). ${ }^{6,31,40}$ Here, we show for the first time that the beneficial effects of TTFC fusion do also apply to full-length gene products, thereby allowing antigen presentation via multiple HLA class I alleles. What is the mechanism by which fusion with a carrier molecule enhances DNA vaccine immunogenicity? In the case of TTFC, a likely explanation is that fusion of genes of interest to TTFC promotes the induction of $\mathrm{CD}^{+} \mathrm{T}$-cell responses through the provision of $\mathrm{CD}^{+}{ }^{+} \mathrm{T}$-cell help via one of the TTFC-encoded "promiscuous" $\mathrm{CD}^{+}{ }^{+}$helper epitopes. ${ }^{6,39}$ Support for this notion comes from prior work demonstrating that a carrier protein needs to be of non-self-origin to improve DNA vaccine immunogenicity and that CD8+ T-cell responses toward a carrier-epitope fusion encoding DNA vaccine are dependent on MHCII-mediated antigen presentation. ${ }^{19}$ In line with this, MHC-II -. / - mice were not able to mount measurable CD8+ T-cell responses after vaccination with either TTFC-E6SH or TTFC E7SH (Supporting Information Fig. 2). However, it is plausible that the presence of helper T-cell epitopes is not the only relevant factor, as-as discussed above-antigen stability correlates with the immunogenicity of DNA vaccines. To assess whether TTFC fusion may increase the accumulation of E7(SH) protein, we transfected HEK293 cells with either E7WT or E7SH or with TTFC-E7WT or TTFC-E7SH and detected E7 protein expression by Western blot analysis 24 $\mathrm{hr}$ after transfection. As shown in Supporting Information Figure 3, E7 accumulation was substantially higher in the TTFC E7(SH)-transfected cells, indicating that the stability of E7 is indeed improved by this fusion.

A remarkable observation in our study is the delayed contraction of the $\mathrm{CD}^{+} \mathrm{T}$-cell responses upon DNA tattoo vaccination with all TTFC-E7 fusion vaccines tested. Conventional $\mathrm{CD}^{+} \mathrm{T}$-cell responses rapidly contract after the peak of the response has been reached, and levels of $\mathrm{CD}^{+} \mathrm{T}$ cells that remain after contraction are generally around $5 \%$ of peak levels. ${ }^{41,42}$ In all our previous studies, immune responses induced by DNA tattoo showed similar kinetics, with contraction being close to complete about 1 week after the peak of the response. ${ }^{16,38}$ In contrast, upon tattooing TTFC-E7encoding DNA vaccines, we consistently observed that contraction of the induced $\mathrm{CD} 8^{+} \mathrm{T}$-cell response was delayed for about 3 weeks, and that the remaining frequencies after 4 weeks were still around $20 \%$ of the initial peak height (Fig. 3). At present, we do not know the cause of this delayed contraction, but the data available suggest that a combination of epitope-intrinsic and epitope-extrinsic factors is required. Specifically, the fact that delayed contraction is observed for TTFC-E7 but not for TTFC-E6 DNA vaccines indicates that epitope identity does play a role. On the other hand, the fact that delayed contraction is observed for TTFC-E7 but not for GFP-E7 ${ }_{49-57}$ DNA vaccines shows that the identity of the carrier also forms a crucial component. Clearly, understanding the molecular basis underlying both the increase in peak height and the improved persistence of $\mathrm{CD}^{+-} \mathrm{T}$-cell responses induced by these fusion vaccines would be highly useful for future DNA vaccine development and will be the focus of our future work.

In conclusion, we have constructed DNA vaccines targeting full-length HPV 16 E6 and E7 with good immunogenicity and safety profiles, by successfully combining strategies to "detoxify" and improve DNA vaccine-encoded antigens. The resulting vaccine format outperformed a vaccine encoding sig/E7(detox)HSP that was recently tested in humans, ${ }^{15}$ providing a strong rationale for clinical evaluation of our vaccine format. We are currently planning to evaluate a combination of TTFC-E7SH- and TTFC-E6SH-encoding constructs, applied via DNA tattoo vaccination, for treatment of HPV 16-positive carcinomas in a Phase I clinical trial.

\section{Acknowledgements}

The vector pNGA4a sig/E7(detox)HSP was kindly provided by Prof. T.C. $\mathrm{Wu}$, Johns Hopkins Medical Institutions, Baltimore, Maryland.

\section{References}

1. Scheurer ME, Tortolero-Luna G, AdlerStorthz K. Human papillomavirus infection: biology, epidemiology, and prevention. Int $J$ Gynecol Cancer 2005;15:727-46.

2. Bosch FX, Lorincz A, Munoz N, Meijer CJ, Shah KV. The causal relation between human papillomavirus and cervical cancer. J Clin Pathol 2002;55:244-65.

3. Roden $\mathrm{R}, \mathrm{Wu}$ TC. How will HPV vaccines affect cervical cancer? Nat Rev Cancer 2006;6:753-63.

4. zur Hausen H. Papillomaviruses and cancer: from basic studies to clinical application. Nat Rev Cancer 2002;2: 342-50.
5. Kutzler MA, Weiner DB. DNA vaccines: ready for prime time? Nat Rev Genet 2008; 9:776-88.

6. Rice J, Ottensmeier $\mathrm{CH}$, Stevenson FK. DNA vaccines: precision tools for activating effective immunity against cancer. Nat Rev Cancer 2008;8: $108-20$.

7. Hung CF, Monie A, Alvarez RD, Wu TC DNA vaccines for cervical cancer: from bench to bedside. Exp Mol Med 2007;39: 679-89.

8. Michel N, Osen W, Gissmann L, Schumacher TN, Zentgraf $\mathrm{H}$, Muller $\mathrm{M}$. Enhanced immunogenicity of HPV 16 E7 fusion proteins in DNA vaccination. Virology 2002;294:47-59.

9. Osen W, Peiler T, Ohlschlager P, Caldeira S, Faath S, Michel N, Muller M,

Tommasino M, Jochmus I, Gissmann L. A DNA vaccine based on a shuffled E7 oncogene of the human papillomavirus type 16 (HPV 16) induces E7-specific cytotoxic $\mathrm{T}$ cells but lacks transforming activity. Vaccine 2001;19:4276-86.

10. Peng S, Ji H, Trimble C, He L, Tsai YC, Yeatermeyer J, Boyd DA, Hung CF, Wu TC. Development of a DNA vaccine targeting human papillomavirus type 16 oncoprotein E6. J Virol 2004;78:8468-76. 
11. Smahel M, Sima P, Ludvikova V, Vonka V. Modified HPV16 E7 genes as DNA vaccine against E7-containing oncogenic cells. Virology 2001;281:231-8.

12. Smahel M, Pokorna D, Mackova J, Vlasak J. Enhancement of immunogenicity of HPV16 E7 oncogene by fusion with $E$. coli beta-glucuronidase. J Gene Med 2004;6: 1092-101.

13. Haigh O, Kattenbelt J, Cochrane M, Thomson S, Gould A, Tindle R. Hepatitis $B$ surface antigen fusions delivered by DNA vaccination elicit CTL responses to human papillomavirus oncoproteins associated with tumor protection. Cancer Gene Ther 2010;17:708-20.

14. Garcia F, Petry KU, Muderspach L, Gold MA, Braly P, Crum CP, Magill M, Silverman M, Urban RG, Hedley ML, Beach KJ. ZYC101a for treatment of highgrade cervical intraepithelial neoplasia: a randomized controlled trial. Obstet Gynecol 2004;103:317-26.

15. Trimble CL, Peng $S$, Kos F, Gravitt $P$, Viscidi R, Sugar E, Pardoll D, Wu TC. A phase I trial of a human papillomavirus DNA vaccine for HPV16+ cervical intraepithelial neoplasia 2/3. Clin Cancer Res 2009;15:361-7.

16. Bins $\mathrm{AD}$, Jorritsma $\mathrm{A}$, Wolkers $\mathrm{MC}$, Hung CF, Wu TC, Schumacher TN, Haanen JB. A rapid and potent DNA vaccination strategy defined by in vivo monitoring of antigen expression. Nat Med 2005;11:899-904.

17. Verstrepen BE, Bins AD, Rollier CS, Mooi P, Koopman G, Sheppard NC, Sattentau Q Wagner R, Wolf $\mathrm{H}$, Schumacher TN, Heeney JL, Haanen JB. Improved HIV-1 specific T-cell responses by short-interval DNA tattooing as compared to intramuscular immunization in nonhuman primates. Vaccine 2008;26:3346-51.

18. Quaak SG, van den Berg JH, Toebes $M$, Schumacher TN, Haanen JB, Beijnen JH, Nuijen B. GMP production of pDERMATT for vaccination against melanoma in a phase I clinical trial. Eur J Pharm Biopharm 2008;70:429-38.

19. Wolkers MC, Toebes M, Okabe M, Haanen IB, Schumacher TN. Optimizing the efficacy of epitope-directed DNA vaccination. $J$ Immunol 2002;168:4998-5004.

20. Howie HL, Katzenellenbogen RA, Galloway DA. Papillomavirus E6 proteins. Virology 2009;384:324-34.

21. McLaughlin-Drubin ME, Munger $\mathrm{K}$. The human papillomavirus E7 oncoprotein. Virology 2009;384:335-44.
22. Cassetti MC, McElhiney SP, Shahabi V, Pullen JK, Le Poole IC, Eiben GL, Smith LR, Kast WM. Antitumor efficacy of Venezuelan equine encephalitis virus replicon particles encoding mutated HPV16 E6 and E7 genes. Vaccine 2004;22:520-7.

23. Ohlschlager P, Pes $M$, Osen W, Durst $M$, Schneider A, Gissmann L, Kaufmann AM. An improved rearranged human papillomavirus type 16 E7 DNA vaccine candidate (HPV-16 E7SH) induces an E7 wildtype-specific T cell response. Vaccine 2006;24:2880-93

24. Munger K, Howley PM. Human papillomavirus immortalization and transformation functions. Virus Res 2002, 89:213-28.

25. Yugawa T, Kiyono T. Molecular mechanisms of cervical carcinogenesis by high-risk human papillomaviruses: novel functions of E6 and E7 oncoproteins. Rev Med Virol 2009;19:97-113.

26. Wolkers MC, Stoetter G, Vyth-Dreese FA, Schumacher TN. Redundancy of direct priming and cross-priming in tumorspecific CD8 + T cell responses. J Immunol 2001;167:3577-84

27. Toebes $M$, Coccoris $M$, Bins A, Rodenko B, Gomez R, Nieuwkoop NJ, van de Kasteele W, Rimmelzwaan GF, Haanen JB, Ovaa H, Schumacher TN. Design and use of conditional MHC class I ligands. Nat Med 2006;12:246-51.

28. Coccoris M, Swart E, de Witte MA, van Heijst JW, Haanen JB, Schepers K, Schumacher TN. Long-term functionality of TCR-transduced T cells in vivo. $J$ Immunol 2008;180:6536-43.

29. Lin KY, Guarnieri FG, Staveley-O'Carroll KF, Levitsky HI, August JT, Pardoll DM, Wu TC. Treatment of established tumors with a novel vaccine that enhances major histocompatibility class II presentation of tumor antigen. Cancer Res 1996;56:21-6.

30. Scardino A, Alimandi M, Correale P, Smith SG, Bei R, Firat H, Cusi MG, Faure O, Graf-Dubois S, Cencioni G, Marrocco J, Chouaib S, et al. A polyepitope DNA vaccine targeted to Her-2/ErbB-2 elicits a broad range of human and murine CTL effectors to protect against tumor challenge. Cancer Res 2007;67:7028-36.

31. Rice J, Elliott T, Buchan S, Stevenson FK. DNA fusion vaccine designed to induce cytotoxic $\mathrm{T}$ cell responses against defined peptide motifs: implications for cancer vaccines. J Immunol 2001;167: 1558-65.
32. Smahel M, Tejklova P, Smahelova J, Polakova I, Mackova J. Mutation in the immunodominant epitope of the HPV16 E7 oncoprotein as a mechanism of tumor escape. Cancer Immunol Immunother 2008; 57:823-31.

33. Wu TC. The role of vascular cell adhesion molecule-1 in tumor immune evasion. Cancer Res 2007;67:6003-6.

34. Boursnell ME, Rutherford E, Hickling JK, Rollinson EA, Munro AJ, Rolley N, McLean CS, Borysiewicz LK, Vousden K, Inglis SC. Construction and characterisation of a recombinant vaccinia virus expressing human papillomavirus proteins for immunotherapy of cervical cancer. Vaccine 1996;14:1485-94.

35. Munger $\mathrm{K}$, Baldwin A, Edwards KM, Hayakawa $H$, Nguyen CL, Owens $M$, Grace M, Huh K. Mechanisms of human papillomavirus-induced oncogenesis. $J$ Virol 2004;78:11451-60.

36. Wolkers MC, Brouwenstijn N, Bakker $\mathrm{AH}$, Toebes M, Schumacher TN. Antigen bias in T cell cross-priming. Science 2004;304: 1314-17.

37. Norbury CC, Basta S, Donohue KB, Tscharke DC, Princiotta MF, Berglund P, Gibbs J, Bennink JR, Yewdell JW. CD8+ T cell cross-priming via transfer of proteasome substrates. Science 2004;304: 1318-21.

38. Bins $\mathrm{AD}$, Wolkers $\mathrm{MC}$, van den Boom MD, Haanen JB, Schumacher TN. In vivo antigen stability affects DNA vaccine immunogenicity. J Immunol 2007;179: 2126-33.

39. Stevenson FK, Rice J, Ottensmeier $\mathrm{CH}$, Thirdborough SM, Zhu D. DNA fusion gene vaccines against cancer: from the laboratory to the clinic. Immunol Rev 2004; 199:156-80.

40. Chaise C, Buchan SL, Rice J, Marquet J, Rouard H, Kuentz M, Vittes GE Molinier-Frenkel V, Farcet JP, Stauss HJ, Delfau-Larue $\mathrm{MH}$, Stevenson FK. DNA vaccination induces WT1-specific $\mathrm{T}$-cell responses with potential clinical relevance. Blood 2008;112: 2956-64.

41. Kaech SM, Wherry EJ, Ahmed R. Effector and memory $\mathrm{T}$-cell differentiation: implications for vaccine development. Nat Rev Immunol 2002;2:251-62.

42. Hand TW, Kaech SM. Intrinsic and extrinsic control of effector T cell survival and memory $\mathrm{T}$ cell development. Immunol Res 2008;45:46-61. 